\title{
Los principales ejemplos de la herencia Jesuítica en la provincia de Madrid y la ciudad de Guadalajara y sus vínculos con la Universidad Complutense
}

\author{
Major examples of Jesuit heritage in the province of Madrid \\ and the city of Guadalajara and links with the University \\ Complutense
}

\author{
GonZALO FERNÁNDEZ*
}

\section{RESUMEN}

Este artículo estudia los primeros edificios de los Jesuitas en Madrid, Alcalá de Henares, Torrejón de Ardoz, y Guadalajara y sus vínculos con la Universidad Complutense donde el Dr. Federico Lara es ilustre profesor.

\section{PALABRAS CLAVE:}

Jesuitas, Alcalá de Henares, Torrejón de Ardoz, Guadalajara, Universidad Complutense.

\section{ABSTRACT}

This article surveys the early buildings of the Jesuits in Madrid, Alcala de Henares, Torrejon de Ardoz, and Guadalajara and its links with the University Complutense where Dr. Federico Lara is illustrious professor.

KEYWORDS:

Jesuits, Alcalá de Henares, Torrejón de Ardoz, Guadalajara, Universidad Complutense.

En la historia de la Compañía de Jesús se distinguen dos grandes épocas. La primera se extiende desde su fundación en 1540 a su extinción en 1773 por el Breve Dominus ac Redemptor del Papa Clemente XIV. Ese Breve se explica por las presiones que las Cortes Borbónicas hacen a Clemente XIV. En el siglo XVIII los grandes enemigos de la Compañía son:

* Universidad de Valencia 
- Rivales institucionales de los jesuitas en el seno de la Iglesia, sobre todo los frailes, parte impulsados por simple envidia parte impelidos por el narcisismo de los jesuitas

- Eclesiásticos de diversos grados que siguen tendencias galicanas y jansenistas

- Las antedichas Cortes Borbónicas que aceptan el regalismo y a cuyos miembros animan los franciscanos y dominicos de sus respectivos países

- Dificultad de adaptarse a la nueva situación por las trece reducciones jesuíticas de Paraguay que pasan a depender de Portugal en 1750

- La llustración anticatólica dentro de la cual destaca la Masonería

La expulsión de los jesuitas de España por Carlos III en 1767 crea una profunda división en la Iglesia Española. Los obispos de Zamora, Segorbe y Mondoñedo se felicitan por la medida. Los arzobispos de Burgos y Orihuela y los obispos de Tarazona, Albarracín y Orihuela apoyan el extrañamiento sin congratularse de manera expresa. El pueblo llano, el obispo de Cuenca Isidoro Carvajal y el Inquisidor General Manuel Quintano y Bonifaz se muestran contrarios. Carlos III destierra al Inquisidor General Quintano al Convento de San Antonio en el pueblo madrileño de La Cabrera al pie de las montañas de Somosierra y el obispo Carvajal ha de comparecer ante el Consejo de Castilla para ser amonestado siendo la primera vez que un obispo ocupa el lugar de los acusados en España.

La segunda época de la historia de la Compañía empieza en 1814 cuando el Papa Pío VII restaura aquella compañía de presbíteros por medio de la Bula Sollicitudo omnium ecclesiarum. La restauración de la Compañía tuvo que vencer las reticencias de los liberales y algunos frailes. En España Fernando VII reestablece la Compañía en 1815 anulando la orden de expulsión que había dado su abuelo Carlos III en 1767. Entre los liberales el poeta Manuel José Quintana critica en 1822 la medida junto a algunas otras que Fernando VII había adoptado durante el sexenio absolutista (1814-1820) como la nueva implantación de los visitadores de las Universidades que habían sido impuestos por el mismo Cisneros en las Constituciones para la Universidad Complutense. Así menciona Quintana en 1822 en el discurso pronunciado con motivo del traslado de la Universidad Complutense desde Alcalá de Henares a Madrid la restauración de aquella Compañía famosa, a quien los reyes han perdonado sus agravios en obsequio de sus intrigas. Asimismo un capuchino, el P. Salvador, predica en la Capilla del Palacio Real contra las grandes alabanzas que se prodigan a la renacida Compañía en menoscabo de las restantes órdenes. Esto le vale el inmediato destierro de la Corte. En 1814 sólo quedan 800 jesuitas anteriores a la extinción de 1773 . Pero crecen rápidamente: en 1820 hay 2.000 y treinta años después la Compañía cuenta con 6.000 miembros. 


\section{ORÍGENES DEL COLEGIO IMPERIAL}

La Compañía de Jesús llega a España muy poco tiempo después de su fundación en 1540. El establecimiento de enseñanza de los jesuitas en la Villa y Corte recibe los nombres de Colegio Imperial de Madrid, Colegio Imperial de la Compañía de Jesús o Colegio de San Pedro y San Pablo de la Compañía de Jesús en la Corte. Su principal benefactora es Da . María de Austria, hija de Carlos I y viuda del Emperador Maximiliano II. El Colegio Imperial se inaugura en 1608, cinco años después de la muerte de la Emperatriz. En 1625 Felipe IV cambia su nombre en los Reales Estudios de San Isidro. La institución no puede conferir grados universitarios por la oposición de las Universidades de Salamanca y Alcalá. Al igual que en todos los centros de enseñanza de los jesuitas se aplica en el Colegio Imperial la Ratio Studiorum que la Compañía impone en 1599 basada en la experiencia de otras escuelas e incluso en las ideas pedagógicas del protestante $\mathrm{Me}$ lanchton que fuesen útiles a los católicos. En el siglo XVII se va creando una biblioteca en los Reales Estudios de San Isidro que es la más importante de Madrid hasta el nacimiento en 1712 de la Biblioteca Pública de Palacio (germen de la actual Biblioteca Nacional).

Los arquitectos jesuitas Pedro Sánchez y Francisco Bautista proyectan la iglesia del Colegio cuyas obras empiezan en 1622. La iglesia se consagra en 1651, aún no terminada por completo, por el Nuncio en Madrid Giulio Rapelosi bajo la advocación de San Francisco Javier. Los últimos detalles se concluyen en 1664. La iglesia sigue el modelo del Gesú de Roma cuyos trabajos son iniciados en 1568 por Giacomo Barozzi da Vignola aunque su portada, la primera portada genuinamente barroca en el sentir de Nithan T. Whitman, se deba a Giacomo della Porta. Finalmente la Casa Grande de Torrejón de Ardoz (Madrid) depende del Colegio Imperial. La Casa Grande se construye en el siglo XV pero pasa a ser propiedad de los jesuitas a inicios del siglo XVII. Los campos de la Casa Grande proporcionan el trigo, vino y aceite que se consumen en el Colegio Imperial cuyas tejas, ladrillos y tinajas se fabrican también en los hornos para cocer barro de la Casa Grande.

\section{LA TRAYECTORIA DEL COLEGIO IMPERIAL}

En el Colegio Imperial son profesores algunos jesuitas eminentísimos:

- El suizo Jean-Baptiste Cysat quien sostiene el carácter regular del movimiento de los cometas

- El flamenco Jan-Karel della Faille al que se debe la determinación del centro de gravedad del sector de un círculo

- El francés Claude Richard estudioso de la Geometría Euclidiana

- El escocés Hugh Semple cuyo libro titulado De mathematicis disciplinis es muy interesante a la hora de conocer la Historia de aquella Ciencia Exacta 
- El español José Zaragoza quien además de ejercer la docencia en el Colegio Imperial enseña Matemáticas a prestigiosas figuras como al Marqués de Leganés D. Diego Felipe de Guzmán y al propio rey Carlos II

También son célebres los actos culturales y representaciones de teatro que hacen los alumnos del Colegio Imperial. Destacan:

- El celebrado en 1629 en cuyo transcurso el P. Nieremberg pronuncia la Prolusión a la doctrina y historia natural y Lope de Vega recita Isagoge a los Reales Estudios de la Compañía de Jesús

- En 1681el estreno de la zarzuela Vencer a Marte sin Marte del jesuita Pedro Fomperosa y Quintana con motivo de la boda de Carlos II con María Luisa de Borbón

Felipe V inaugura el Real Seminario de Nobles dentro de los Reales Estudios de San Isidro con el propósito de crear un grupo de estudiantes aventajados. Cuando Carlos III expulsa a los jesuitas en 1767 los Reales Estudios de San Isidro se cierran. Vuelven a abrirse tres años más tarde bien que su titularidad no pertenezca a la Compañía. Dado que Carlos III nombra los maestros los Reales Estudios de San Isidro se convierten en un foco de regalismo. Su iglesia pasa a ser Colegiata. Su advocación cambia de San Francisco Javier a San Isidro Labrador. En su Altar Mayor se depositan los restos mortales del Santo Patrón de la Villa y Corte y de su esposa Santa María de la Cabeza. La Casa Grande pasa ser propiedad de Juan de Aguirre en 1767 y en 1805 es adquirida por el conde de Fuentes quien pertenece a la familia aragonesa de los Pignatelli.

Los jesuitas vuelven a ocuparse de los Reales Estudios de 1816 a 1834 con el paréntesis del Trienio Constitucional (1820 - 1823) aunque no de la Colegiata de San Isidro. Esta última se transformará en 1885 en la Catedral de Madrid hasta la consagración de La Almudena en 1993. Fernando VII devuelve también la Casa Grande de Torrejón de Ardoz a la Compañía. Es terrible para los Reales Estudios de San Isidro la matanza de regulares que tiene lugar el 17 de julio de 1834. Ese día supone el eco en España de la Revolución de 1830 en Francia de la misma manera que el Trienio Constitucional y la Primera República de 1873 son las recepciones españolas de la Revolución Francesa de 1789 (el Trienio Constitucional) y la Comuna de París de 1871 (la Primera República). El tumulto empieza en 1834 cuando una epidemia de cólera morbo llega a Madrid donde se desarrolla con intensidad terrible la noche del 15 de julio y las masas empiezan a excitarse con la acusación de que agentes carlistas habían envenenado las fuentes.

De los jesuitas que viven en los Reales Estudios son asesinados el 17 de julio de 1834:

- Presbíteros PP. Juan Artigas, José Fernández y Francisco Sanz

- Diáconos José Elola y José Urreta

- Subdiáconos Domingo Barrau, José Garnier, José Sancho y Pedro Demont 
Los principales ejemplos de la herencia Jesuítica en la provincia de Madrid...

- Minoristas Fermín Barba, Martín Buxons, Manuel Ostolaza, Juan Ruedas y Vicente Gogorza

A su vez sufren heridas de consideración:

- Padre Celedonio Unanúe

- Hermanos estudiantes Sabas Trapiella y Francisco Sanz

- Hermano coadjutor Julián Acosta

\section{LOS NUEVOS DESTINOS DE LOS REALES ESTUDIOS DE SAN ISIDRO Y SU BIBLIOTECA Y LA CASA GRANDE}

En 1835 los jesuitas son de nuevo expulsados de España por el gobierno que encabeza el conde de Toreno. Los Reales Estudios de San Isidro pasan a denominarse Estudios Nacionales. En 1844 se adscriben a la Universidad Literaria de Madrid como Instituto de Enseñanza Media hasta la actualidad. El bibliófilo Bartolomé José Gallardo se ocupa de transferir los manuscritos de la antigua Biblioteca de los Reales Estudios de San Isidro a la de las Cortes dado que en 1834 recibe Gallardo el nombramiento de bibliotecario de las Cortes de la Nación. Esos mismos manuscritos son hoy posesión de la Real Academia de la Historia. En 1844 el resto de la Biblioteca de los Reales Estudios se traslada a la de la Universidad Literaria de Madrid que sólo en 1850 recibe el título de Central. En principio la Biblioteca de la Universidad Literaria de Madrid se halla en la Facultad de Filosofía también ubicada en el antiguo Noviciado de los Jesuitas. Su Bibliotecario Mayor interino es en 1845 el magnífico historiador Vicente de la Fuente hoy tan injustamente olvidado. La Universidad Literaria de Madrid recibe otras herencias del Colegio Imperial: un botamen farmacéutico y un retrato anónimo del P. Juan de Mariana que se halla hoy en el Decanato de la Facultad de Filosofía.

La Biblioteca del Colegio Imperial era particularmente rica en libros de Ciencias. Las Bibliotecas de la Universidad Complutense poseen hoy los ejemplares de las siguientes obras que poseían los jesuitas en aquel establecimiento:

- Dialogo della descrittione teorica et practica de gli horologi solari de GianBattista Vimercato (Ferrara, 1565)

- Cronología hoc est temporum de Gerardo Mercator (Colonia, 1569)

- Ad vitellionem paralipomena de Johannes Kepler (Francfort del Meno, 1604)

- Logarithmorum ad totidem numeros rotundos del propio Kepler (Marburgo, 1624)

- Le operationi del compasso geometrico de Galileo Galilei (Bolonia, 1656)

- Aritmética Universal de José Zaragoza (Valencia, 1669) 
- Esphera en comun celeste y terraquea del mismo Zaragoza (Madrid, 1675)

- Philosophiae Naturalis principia matemática de Isaac Newton en su primera edición (Londres, 1687)

- Apparatus chronologicus et geographicus de Bernard Lamy (Venecia, 1735)

- Défense de la chronologie fondée sur les monuments de I histoire ancienne contre le sisteme chronologique de M. Newton por Nicolás Freret (París, 1758)

En 1835 la Casa Grande de Torrejón de Ardoz vuelve a la familia de los Pignatelli quienes la conservan hasta 1940. La Casa Grande pasa a ser sede de una empresa agropecuaria y casa-cuartel de la Guardia Civil. En 1973 la adquiere el empresario cordobés Rafael Onieva. Onieva la transforma en un importante centro hotelero, gastronómico y cultural. Rafael Onieva adquiere y amplía la riquísima colección de iconos del militar zarista Sergi Otzoup. La instala en el antiguo horno para cocer barro de la Casa Grande del que en su tiempo salieron las tejas, ladrillos y tinajas del Colegio Imperial.

\section{EL NOVICIADO DE LOS JESUITAS EN MADRID (CALLE ANCHA DE SAN BERNARDO)}

Hacia 1600 Dª . Ana Félix de Guzmán, marquesa de Camarasa, compra una finca en la calle ancha de San Bernardo y se la regala a los jesuitas. Éstos deciden establecer allí su Noviciado. En 1605 se inaugura la iglesia del Noviciado. En 1612 Dª . Ana Félix de Guzmán es enterrada en aquel santo lugar. Muchos otros nobles siguen su ejemplo y piden que sus restos se sepulten en esa iglesia. El término de las obras del Noviciado Jesuítico sólo acaece a mediados del siglo XVII.

En 1767 Carlos III cede el Noviciado con su iglesia a la Venerable Congregación de Sacerdotes Misioneros del Salvador del Mundo, vulgo Padres del Salvador, quienes han de abandonarlos en 1829 pues Fernando VII quiere devolverlos a los jesuitas. La nueva expulsión de los jesuitas en 1835 hace que allí se instale un cuartel de Ingenieros Militares. En 1842 se empiezan a instalar en dicho recinto las facultades de la Universidad Complutense que se trasladan a Madrid desde Alcalá de Henares para constituir en la Villa y Corte la Universidad Literaria. Aquel año se emplazan la sede del Rector, las dependencias administrativas y las Facultades de Teología, Filosofía y Jurisprudencia. En 1845 se añaden las de Medicina y Farmacia. Asimismo se publican los primeras medidas legales de la Universidad Literaria de Madrid con estas disposiciones:

1) Ser la única que puede conferir el grado de Doctor

2) Sometimiento a los principios de uniformidad, centralización y secularización comunes a todas las Universidades del Reino bien que la de Madrid sea su eje vertebrador 
Los principales ejemplos de la herencia Jesuítica en la provincia de Madrid...

3) Establecimiento de planes de estudio, métodos docentes y nuevas formas de exámenes

4) Nombramiento del Rector Magnífico por la Reina

5) Creación de un cuerpo único de profesores al que se accede por oposición

6) Control por el Gobierno de programas y libros de texto

7) Distinción de tres tipos de estudios:

- Facultades Mayores (Filosofía, Teología, Jurisprudencia, Medicina y Farmacia)

- Estudios Superiores destinados a la colación del grado de Doctor

- Estudios Especiales donde se engloban las carreras profesionales, Ingenierías y la carrera de Farmacia

La reforma del edificio se encarga a Francisco Javier Mariátegui, antiguo discípulo de Juan de Villanueva, quien pretende:

- Dignificar la fachada con poco gasto a base de arcos de descarga y pilastras adosadas

- Romper la monotonía del alero del viejo noviciado con un frontón

En 1842 Francisco Javier Mariátegui empieza a derruir la iglesia para hacer el Paraninfo de la Universidad Central por medio del desalojo de las tumbas, la demolición de las torres y la metamorfosis de la antigua planta en forma de cruz latina de la iglesia en una nueva planta elipsoidal. Mariátegui muere en 1844. Le sustituye Francisco Pascual y Colomer quien sigue las pautas de su antecesor al añadir la fachada neoclásica que hoy se ve. La planta y el alzado del Paraninfo se concluyen en 1852 con las siguientes dimensiones: 36 metros y 12 centímetros de longitud, 15 metros y 40 centímetros de anchura y 18 metros con 38 centímetros de altura. El pintor catalán Joaquín Espalter y Rull decora el techo con 10 figuras alegóricas y 31 retratos que se distribuyen en:

- Retrato de Isabel la Católica

- Retrato de Isabel II

- 20 retratos de hombres célebres

- 9 retratos de los fundadores de las Universidades españolas

El Paraninfo se inaugura en la apertura oficial del año académico 18541855 aunque sin terminar el lucernario. El Paraninfo de la Complutense sigue allí y comparte el Caserón de San Bernardo con el Consejo Escolar del Estado instalado en 1956 como Consejo Nacional de Educación, el Instituto de España que se establece en 1968 y la Real Academia de Doctores que en dichio emplazamiento se afinca en 1969. 


\section{LA COMPAÑÍA DE JESÚS EN ALCALÁ DE HENARES}

Los compañeros de San Ignacio llegan a Alcalá de Henares en 1546 por los estímulos que la Emperatriz María, la Reina Madre de Portugal Juana y la antigua aya de Felipe II dan al P. Francisco Villanueva S.J. El P. Villanueva estudiaba en el Colegio Mayor de San Ildefonso de la Universidad Complutense desde 1543. La Familia de los Mendoza y el Duque de Gandía (futuro San Francisco de Borja) ayudan al P. Villanueva a comprar un solar en Alcalá, en concreto en la Calle Libreros, donde hoy se alzan la Parroquia de Santa María la Mayor y la Facultad de Derecho de la Universidad de Alcalá de Henares que aprovechan las viejas Iglesia y Colegio Máximo de la Compañía de Jesús. No obstante, el P. Villanueva construye en principio una Iglesia y un Colegio Máximo más pequeños derribados a comienzos del siglo XVII para dar paso a los actuales. La primera se debe al arquitecto jesuita Bartolomé de Bustamante. Se consagra en 1562. El primer Colegio Máximo se acaba antes. Lo erige el mismo P. Villanueva siguiendo las directrices de San Ignacio de Loyola. Allí estudian los eximios escritores jesuitas PP. Juan de Mariana y Juan Eusebio Nieremberg. La Parroquia de Santa María la Mayor y la Facultad de Derecho de la Universidad de Alcalá son dos joyas del barroco jesuítico en la ciudad complutense.

\section{LA IGLESIA DE LA COMPAÑÍA (HOY PARROQUIA DE SANTA MARÍA LA MAYOR)}

Se inicia en 1602 y se termina en 1625 bajo el mecenazgo de doña Catalina de Mendoza y Mendoza. Su traza actual se debe al arquitecto conquense Francisco de Mora aunque su ejecución corre a cargo de Gaspar Ordóñez y Valentín Ballesteros. Francisco de Mora había sido discípulo de Juan de Herrera. Es el arquitecto del Duque de Lerma y su hijo el Duque de Uceda (validos ambos de Felipe III). A Francisco de Mora se deben el Palacio de Uceda en Madrid, la Iglesia de San José en Ávila y en Lerma el Palacio Ducal y los seis conventos. Para construir la Iglesia de la Compañía en Alcalá Francisco de Mora se inspira en la Iglesia del Gesú de Roma.

Juan Gómez de Mora (sobrino de Francisco de Mora) diseña la fachada de la Iglesia de la Compañía en Alcalá. Gómez de Mora adopta como referencia la portada de la Iglesia de Santiago de los Incurables de la Ciudad Eterna que principia Francesco da Volterra y concluye Carlo Maderno entre 1598 y 1602. Sin embargo es Valentín Díez Arias el ejecutor de la fachada de la Iglesia Jesuítica de Alcalá, siguiendo los planos de Gómez de Mora. En la decoración de la fachada destacan las estatuas del escultor portugués Manuel Pereira que las hace en 1624. Representan a San Pedro y San Pablo (intercolumnios del primer cuerpo) y San Ignacio de Loyola y San Francisco Javier (intercolumnios del cuerpo superior). En las entradas laterales figura por repetido el escudo heráldico de los Mendoza. 
Los principales ejemplos de la herencia Jesuítica en la provincia de Madrid...

La planta de la Iglesia de la Compañía en Alcalá de Henares es de cruz latina. Tiene capillas comunicadas entre sí y una cúpula no trasdosada. De 1618 a 1629 Francisco Bartolomé levanta el Retablo del Altar Mayor. Los lienzos originales del Retablo eran de Ángelo Nardi pero se destruyen durante la Guerra Civil. Tras la expulsión de los jesuitas de España por Carlos III en 1767, la Iglesia de la Compañía se trasforma en la Parroquia de Santa María la Mayor cuyo cometido sigue desempeñando en la actualidad.

\section{EL PRODIGIO Y LA CAPILLA DE LAS SANTAS FORMAS}

En mayo de 1597 un desconocido entrega al P. Juan Juárez S.J. 26 Sagradas Formas a las que salva de su profanación a manos de moriscos. Esas Formas se mantienen incorruptas en la Iglesia de la Compañía y luego Parroquia de Santa María la Mayor hasta 1936. En 1620 la autoridad eclesiástica permite su culto que dura hasta el antedicho 1936. En 1687 los alcalaínos erigen la Capilla de las Santas Formas dentro de la Iglesia de la Compañía por suscripción popular. La nueva Capilla tiene planta de cruz griega. En 1699 Juan Vicente Rivera decora la cúpula de la Capilla de las Santas Formas con pinturas al óleo. El rastro de las Santas Formas se pierde en 1936. Ante la persecución de la Iglesia en la España Republicana los presbíteros alcalaínos Pedro García Izcaray, Eduardo Ardiaca y Pablo Herrero Zamorana ocultan las Santas Formas por temor a profanaciones pero los tres sacerdotes mueren en el transcurso de la contienda fratricida.

\section{EL COLEGIO MÁXIMO}

Andrés Ramírez erige una fachada de ladrillo en 1620. De 1660 a 1690 Melchor de Bueras edifica la fachada actual a base de ventanales que se cierran por medio de rejas. Una vez expulsados los jesuitas de España en 1767, Carlos III encarga a Ventura Rodríguez la transformación del antiguo Colegio Máximo de la Compañía de Jesús. El monarca decide instalar allí la Universidad Complutense que se desgaja de su lugar habitual en el Colegio de San Ildefonso. Ventura Rodríguez reforma la fachada de Melchor de Bueras adaptándola al gusto neoclásico a base de eliminar parte de su decoración barroca. Añade un nuevo patio y crea una escalera imperial.

En 1797 la Universidad Complutense vuelve a su primer hogar en el Colegio Mayor de San Ildefonso. El Colegio Máximo pasa a ser cuartel. En 1827 Fernando VII lo devuelve a los jesuitas. En 1835 el conde de Toreno expulsa nuevamente a la Compañía de España. Un año después acaece el traslado definitivo de la Universidad Complutense desde Alcalá a Madrid. Entonces el Colegio Máximo de los jesuitas pasa a ser el cuartel de Mendigorría. La Universidad de Alcalá de Henares vuelve a la vida en 1977 bien que privada de su viejo nombre de Complutense. En la década de 1980-1990 la renacida Universidad se beneficia de ad- 
ministraciones socialistas simultáneas en el Estado, la Autonomía Madrileña y el Ayuntamiento de Alcalá sobre todo con los regidores D. Arsenio Lope Huerta y D. Florencio Campos Corona. Esas personas e instituciones favorecen al Rector D. Manuel Gala Muñoz con un magnífico plan por el que el Ministerio de Defensa cede a la Universidad una serie de antiguos Colegios usados hasta entonces como cuarteles. Uno de ellos es el cuartel de Mendigorría en 1990 que se convierte dos años más tarde en la Facultad de Derecho gracias al arquitecto D. Antonio Fernández Alba.

\section{LA BIBLIOTECA DEL COLEGIO MÁXIMO}

Era muy importante en libros impresos y manuscritos. En 1767 Carlos III ordena que los libros impresos de las bibliotecas de los jesuitas expulsos se entreguen a las Universidades en los lugares donde coexistieran antiguas casas de jesuitas con esos centros de enseñanza superior o en su defecto al obispo de la diócesis para que forme bibliotecas públicas uniendo las antiguas bibliotecas jesuíticas a las de prelados fallecidos y otros legados. Con los impresos se formaría un Archivo General de Temporalidades.

En 1767 se piensa unir la biblioteca del Colegio Máximo a la del Colegio Mayor de San Ildefonso. Ante las protestas del resto de la Universidad Complutense se crea una biblioteca propia de la Universidad a la que se da el nombre de Biblioteca Complutense con los libros impresos del Colegio Máximo. La Biblioteca Complutense sería una parte de la Universidad homónima que se trasladaría del Colegio de San Ildefonso al Colegio Máximo reformado por Ventura Rodríguez. Por el contrario Carlos III dispone que los manuscritos del Colegio Máximo se envíen al Archivo General de Temporalidades.

En el recuento que entonces se lleva a cabo de esos fondos impresos y manuscritos se aportan los mejores datos sobre la biblioteca del Colegio Máximo. Allí se dice que:

— Los jesuitas tenían unos 16.000 ó 17.000 libros

- Son más ricos los fondos de los siglos XV y XVI que los del XVII y XVIII

- Existen duplicados principalmente de libros de los Santos Padres y escritores eclesiásticos o morales

- La mayoría están escritos en latín aunque existen también libros franceses, italianos, portugueses y castellanos

- Hay uno o dos libros en alemán, uno en árabe y ninguno en inglés

A su vez se registran dentro de los manuscritos:

- Bulas auténticas

- Libros membranáceos manuscritos 
- Libros chinos

- 1 manuscrito arábigo

- 1 manuscrito griego

- Libros de estampas

En 1797 la Biblioteca Complutense se une con la del Colegio Mayor de San IIdefonso y en su interior alberga cuatro salas:

- Primera: libros de Teología, Derecho y Humanidades

- Segunda: Historia y ediciones de la Biblia

- Tercera: Geografía, Historia Natural y Miscelánea

- Cuarta: los papeles del Cardenal Cisneros que se hallaban en el Colegio Mayor de San Ildefonso desde su fundación

Con el traslado de la Universidad Complutense a Madrid en 1836 esta Biblioteca se traslada a la Villa y Corte de 1841 a 1843. Se instala en las Nuevas Salesas mientras se remodela el antiguo edificio del Noviciado de los jesuitas en la Calle Ancha de San Bernardo para convertirlo en la nueva sede de la Universidad Madrileña.

\section{ANTIGUOS ESTABLECIMIENTOS JESUÍTICOS EN LA CIUDAD DE GUADALAJARA}

Los jesuitas llegan a la ciudad española de Guadalajara a finales del siglo XVI. Pero la Compañía de Jesús sólo decide construir un Colegio en la capital de la Alcarria en 1619 a instancias del linaje de los Lasarte y más en concreto de D. Diego de Lasarte y su mujer Da․ Mencía. En 1631 se inauguran el Colegio y convento anexo. La iglesia se consagra en 1647. Colegio e iglesia se dedican a la Santísima Trinidad. El primer Rector del Colegio de la Trinidad es el P. Hernando Pecha S.J.

EI P. Pecha había estudiado en el Colegio Máximo de los jesuitas en Alcalá de Henares. Por desgracia no imprime sus obras en vida. Se conserva manuscrito un Tratado del P. Pecha cuyo asunto es la primacía de la Archidiócesis de Toledo sobre los restantes obispados de España. Ese escrito ha de referirse a la polémica que en el siglo XVII divide a la Iglesia Española en torno a cuál Archidiócesis debe ser la Primada: si Toledo o Santiago de Compostela. Tampoco se editan un Comentario al Cronicón de Julián Pérez y el Parecer de don Tomás Tamayo sobre la ciudad complutense de Pecha. Del mismo autor se ha perdido una Vida de doña Ana de Mendoza duquesa del Infantado.

Sólo en 1977 la benemérita Institución Cultural Marques de Santillana, dependiente de la Diputación Provincial de Guadalajara, da a la imprenta la Historia de Guadalaxara y cómo la Religión de San Gerónymo fue fundada y restaurada por 
sus ciudadanos de Hernando Pecha. Este libro se había escrito hacia 1632 y se conservaba inédito en el manuscrito №. 1.756 de la Biblioteca Nacional de Madrid. La Historia de Guadalaxara de Pecha se divide en cuatro partes que se dedican:

- La primera a la fundación de la Orden Jerónima

- La segunda a los personajes eclesiásticos de la capital de la Alcarria

- La tercera a la ciudad de Guadalajara, su organización y biografías de sus personajes relevantes

- La cuarta al linaje de los Mendoza

La Historia de Guadalaxara del P. Pecha tiene el error de postular que la Complutum romana es Guadalajara y no Alcalá de Henares. Pecha sigue a un historiador alcarreño del siglo XVI que se llama Francisco Medina y Mendoza. De Medina y Mendoza sólo se publica la Vida del Cardenal D. Pedro Gómez de Mendoza. Permanecen inéditos los Anales de la Ciudad de Guadalajara, la Historia del rey Don Enrique IV, Genealogía de la Casa Mendoza, Nobleza y títulos de la Casa Mendoza y la Vida del Cardenal D. Fray Francisco Ximénez de Cisneros. El historiador del siglo XVII Alonso Núñez de Castro publica en Madrid en 1653 la Historia eclesiástica y seglar de la muy noble y leal ciudad de Guadalajara donde se atribuye a Medina y Mendoza la más antigua identificación de la Complutum romana con Guadalajara.

\section{LA EXPULSIÓN DE LOS JESUITAS DE ESPAÑA EN 1767 Y SU IMPACTO EN EL COLEGIO, IGLESIA Y BIBLIOTECA DE LA TRINIDAD}

El extrañamiento de los jesuitas en 1767 motiva que el Colegio se venda a particulares que lo habilitan como viviendas y locales comerciales. En esos mismos años los ilustrados destruyen la Iglesia mudéjar de San Nicolás y erigen allí un teatro. La Parroquia de San Nicolás era la sede del arciprestazgo arriacense que depende del obispado de Sigüenza. Los días 7 y 8 de septiembre de 1770 se traslada la Parroquia de San Nicolás a la antigua Iglesia de la Compañía de Jesús a la que se cambia su advocación por la de Parroquia de San Nicolás según una nota histórica intercalada entre las partidas del Libro de Bautismos fechada el 15 de septiembre de aquel año que alude a la traslación secreta el 7 de septiembre y a la pública del día siguiente (Archivo parroquial de San Nicolás el Real, $n^{\circ} 4$, fol. $123 v$ a 130r). El coliseo dieciochesco pasa a ser el Teatro Municipal de Guadalajara que se derriba en 1930. El edificio actual del Banco de España se levanta en su emplazamiento'.

1 Agradezco a D. Antonio Herrera Casado el envío por correo electrónico de estos preciosos datos. El amable e-mail de don Antonio dice:

He encontrado estos documentos, con fechas, que hablan del traslado de la vieja parroquia de San Nicolás, edificio que presumimos mudéjar, a la iglesia de la Compañía. Son estos: 
Los principales ejemplos de la herencia Jesuítica en la provincia de Madrid...

Tras la expulsión de la Compañía de España y sus Indias la biblioteca de los jesuitas alcarreños pasa a poder del obispo de Sigüenza quien la dona a la Universidad de Sigüenza. Allí permanecen hasta la extinción de la Universidad de Sigüenza en 1837. De Sigüenza vuelven a Guadalajara donde constituyen el fondo más antiguo de la Biblioteca Pública Municipal que se instala en parte del antiguo Convento de la Piedad que Mendizábal había expropiado a sus legítimas dueñas franciscanas con motivo de la Desamortización.

\section{BIBLIOGRAFÍA}

BERNAL GÓMEZ, B. La política Universitaria del Cardenal Cisneros en L. J. MORENO, A. JIMÉNEZ GARCÍA Y M. OCAÑA OCAÑA: La Universidad Complutense cisneriana: impulso filosófico,científico y literario,siglos XVI y XVII, Colección Philosophica Complutensia no. 7, Editorial Complutense, Madrid, 1996.

GARRIDO, F., ¡Pobres Jesuitas! Origen, doctrinas, máximas, privilegios y visicitudes de la Compañía de Jesús desde su fundación hasta nuestros días, seguidas de la Monita Secreta, o Instrucciones ocultas de los jesuitas, por primera vez publicadas en castellano. $2^{\mathrm{a}}$ ed., Madrid. http://www.filosofia.org/aut/fgt/pobres17.htm.

HERRERA CASADO, A. Guadalajara una ciudad que despierta. Guadalajara, 1991

LÓPEZ TRUJILLO, M. A. y GARCIAA-RISCO VIGARA, M. C. La comisión de monumentos de Guadalajara (1835 - 1939). Breve reseña histórica e inventario de fuentes, Alcalá de Henares, $h$ ttp://www2.uah.es/histant/resena.htm.

LÓPEZ TRUJILLO, M. A. y GÓMEZ-PANTOJA, J. Los inicios de la arqueología moderna en Guadalajara: dos notas sobre yacimientos poco conocidos, Alcalá de Henares, http://www2.uah.es/histant/inicios4d.htm

LÓPEZ TRUJILLO, M. A. y GARCÍA-RISCO VIGARA, M. C. La comisión de monumentos de Guadalajara (1835 - 1939). Breve reseña histórica e inventario de fuentes, Alcalà de Henares, http://www2.uah.es/histant/resena.htm.

LÓPEZ TRUJILLO, M. A. Un inédito inventario arqueológico, histórico y artístico. La comisión de monumentos de Guadalajara (1844-1845), Alcalá de Henares, http://www2.uah. es/histant/inventario4a.htm.

MENÉNDEZ PELAYO, M. Historia de los Heterodoxos Españoles. Tomo VII. Heterodoxia en el siglo XIX. Alicante, http://www.cervantesvirtual.com/FichaObra.html?Ref=836.

(Documento original: Archivo parroquial de San Nicolás el Real, de Guadalajara, Nota histórica intercalada entre las partidas del Libro de Bautismos $n^{\circ}$ 4, fol. $123 \mathrm{~V}$ a 130r. Guadalajara, 15 de septiembre de 1770) [123v] Parroquia del Real de San Nicolás. Su Traslación secreta en 7 de Septiembre de 1770

En el día siete del mes de Septiembre del año de mil setecientos setenta, se trasladó la Parroquia de San Nicolás de esta Ciudad de Guadalaxara a la Iglesia que fue antes de los Regulares, llamados Jesuitas de la Compañia de Jesús, expatriados de todos los dominios de nuestro católico monarca [124r] Parroquia del Real de San Nicolás. Su Traslación Pública en 8 de Septiembre de 1770 (que Dios guarde) y desde el día de dicha traslación se mandó llamar, en virtud de Real Decreto, con el dictado de Real Parroquia de San Nicolás, cuya traslación se practicó en la forma siguiente.

En el día quatro de Abril del año de Mil setecientos sesenta y siete fueron extrañados de todos los dominios de España, Indias e Islas adyacentes de nuestro Católico monarca, todos los Regulares de la Compañia [124v] Ilamada de Jesús, con cuyo motivo quedaron vacantes sus Iglesias. Pidió el Consejo informe a todas las Ciudades [preguntando] para qué Iglesias serían a propósito las de dichos Jesuitas, y así lo practicaron. 
MIGUEL ALONSO, A. Los bienes de la Compañía de Jesús incautados en Madrid en 1767 y 1835, y conservados en la Universidad Complutense, Madrid, http://www.cervantesvirtual.com/servlet/SirveObras/01367399871214942243679.

QUINTANA, M. J. Discurso pronunciado en la Universidad Central el día de su instalación: (7 de Noviembre de 1822). Madrid, http://www.cervantesvirtual.com/servlet/SirveObras/scclit/12715065330169392976846/index.htm.

ROGIER, L.J., BERTIER DE SAUVIGNY, G. DE y HAJJAR, J. Nueva Historia de la Iglesia. Tomo IV. De la llustración a la Restauración. Trad. española de J. VALIENTE MALLA. Madrid, 1977.

SANTOS ARÁMBURU, A. La Biblioteca Histórica Marqués de Valdecilla de la Universidad Complutense: un centro de apoyo a la investigación y la docencia, Madrid, http://www.ucm.es/BUCM/revistas/ghi/02144018/articulos/CHMO0606110141A.PDF.

SANTOS ARÁMBURU, A. La Colección de libros de Matemáticas de la Biblioteca Histórica de la Universidad Complutense, Madrid, http://www.ucm.es/BUCM/foa/ exposiciones/mat2006/00.html.

TÜCHLE, H. y BOUMAN, C. A. Nueva Historia de la Iglesia. Tomo III. Reforma y Contrarreforma. Trad. española de A-P. SÁNCHEZ PASCUAL. Madrid, 1966.

VARIOS AUTORES. Historia General de España. La compuesta, enmendada y añadida por el Padre Mariana con la continuación de Miniana; completada con todos los sucesos que comprenden el escrito clásico sobre el reinado de Carlos III, por el Conde de Floridablanca, la Historia de su levantamiento, guerra y revolución, por el Conde de Toreno y la de nuestros días por Eduardo Chao. Tomos IV y V. Madrid. http://books.google.es/books?as_brr=3\&q=Mariana+\%22Historia+general+de+Espa\%C3\%B1a\%22\&btnG=Buscar+libros. 